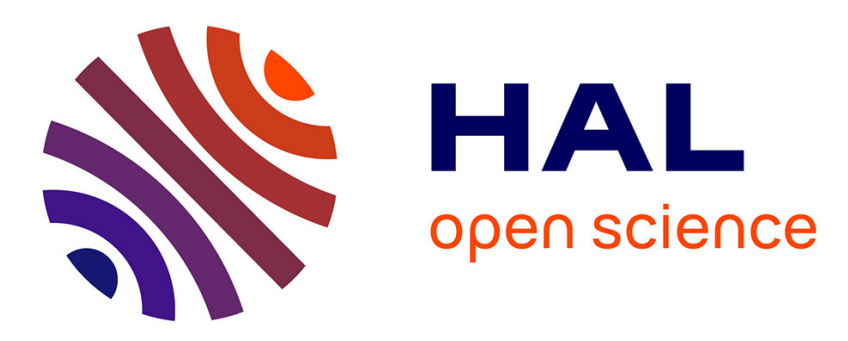

\title{
Atomic level description of the protecting effect of osmolytes against thermal denaturation of proteins
}

Stefano Pieraccini, Luigi Burgi, Alessandro Genoni, Anna Benedusi, Maurizio Sironi

\section{- To cite this version:}

Stefano Pieraccini, Luigi Burgi, Alessandro Genoni, Anna Benedusi, Maurizio Sironi. Atomic level description of the protecting effect of osmolytes against thermal denaturation of proteins. Chemical Physics Letters, 2007, 438 (4-6), pp.298-303. 10.1016/j.cplett.2007.03.007 . hal-02196454

\section{HAL Id: hal-02196454 \\ https://hal.univ-lorraine.fr/hal-02196454}

Submitted on 27 May 2020

HAL is a multi-disciplinary open access archive for the deposit and dissemination of scientific research documents, whether they are published or not. The documents may come from teaching and research institutions in France or abroad, or from public or private research centers.
L'archive ouverte pluridisciplinaire HAL, est destinée au dépôt et à la diffusion de documents scientifiques de niveau recherche, publiés ou non, émanant des établissements d'enseignement et de recherche français ou étrangers, des laboratoires publics ou privés. 


\title{
Atomic level description of the protecting effect of osmolytes against thermal denaturation of proteins
}

\author{
Stefano Pieraccini, ${ }^{a}$ Luigi Burgi, ${ }^{\text {a }}$ Alessandro Genoni, ${ }^{a}$ Anna Benedusi, ${ }^{\text {b }}$ Maurizio Sironi ${ }^{\text {a,c, },}$
}

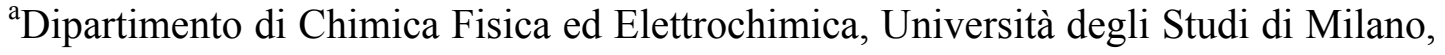
Via Golgi 19, 20133, Milano, Italy.

${ }^{\mathrm{b}}$ Giuliani s.p.a. Via Palagi 2, 20100, Milano.

${ }^{\mathrm{c}}$ Center of Biomolecular Interdisciplinary Studies and Industrial Applications (CISI), Via Fantoli 16/15, 20138, Milano, Italy.

\begin{abstract}
The protecting effect of the osmolyte molecule taurine against thermal denaturation of the protein Chimotripsin Inhibitor 2 was modelled using Molecular Dynamics simulations. The protein was simulated in denaturing conditions at different taurine concentrations. Analysis of the molecular details of its behaviour shows that the protective effect of the osmolyte is concentration dependent. Moreover the influence of taurine on the solvent structure was studied. A concentration dependent ordering effect of taurine on water molecules emerges from solvent structure analysis and is well correlated to the protecting effect observed. Based on these observations an interpretation of the osmoprotective effect is proposed.
\end{abstract}

Osmoprotectants are small highly soluble molecules present in all organisms. They were discovered in connection with the osmotic stress response of some living forms which can survive in extremely harsh life conditions, such as those found in desert or icy environments. Afterwards, it became evident that they can play a role as chemical chaperones, i.e. they can protect the native structure of a protein from adverse chemical and/or environmental agents which could otherwise denature the protein. Osmoprotectants have no net charge at physiological $\mathrm{pH}$, and are non-toxic at high intracellular 
concentrations, so that they do not perturb the protein machinery and hence they are also called 'compatible solutes' ${ }^{1}$. Different mechanisms for osmoprotectant action have been proposed so far, involving a direct interaction with the protein surface or an indirect effect through an action on the solvent molecules. The specific mechanism could also be dependent on the osmoprotectant concentration. Since it is known that only a limited number of osmoprotectant molecules protects a large variety of proteins, it has been proposed that their mechanism of action could be quite general and that this could be the result of a 'genetic simplicity'2. As the elucidation of the protective effect at molecular level is difficult to achieve by experimental methods, molecular dynamics (MD) calculations can be of great value. Recently, the molecular mechanism of stabilization of a protein, namely chymotrypsin inhibitor 2 (CI2), induced by the trimethylammine N-oxyde (TMAO) in presence of the well known denaturant urea, was investigated ${ }^{3}$, while a theoretical study of the action of osmoprotectants against protein thermal denaturation is still lacking. In this letter we present a MD study on the capacity of 2 -aminoethansulphonic acid (taurine, Fig. 1$)^{2}$ to protect CI2 from a thermal denaturation. The CI2 protein was chosen because a great deal of experimental and theoretical data is available on its folding-unfolding mechanism ${ }^{4}$. Furthermore, extensive simulations have shown that higher temperatures enhance the rate of unfolding without changing the unfolding $\operatorname{path}^{5}$. This fact allows to perform simulations at a temperature higher than the protein melting point $(373 \mathrm{~K})$, that permits a wider exploration of the phase space and the observation of unfolding phenomena in an accessible computer time, without loosing physical reality of the system. Our goal was to gain more insight in the osmolyte action at the atomic level by simulating systems comprising $\mathrm{CI} 2$ and a water solution containing taurine at different concentrations.

The CI2 crystal structure (PDB ID: 1 YPC) ${ }^{6}$ was used as the initial protein geometry. A control simulation consisting of the protein solvated with water molecules and three simulations with the addition of an increasing number of taurine molecules (50, 100 and 200 molecules) were carried out. In all the systems the protein was solvated in a water box of $4.68 \mathrm{~nm}$ side length, adding two chloride ions to neutralise the system. The systems with the osmoprotectant molecules were prepared 
with 50, 100 or 200 taurine molecules randomly positioned in the box before solvation, in order to simulate solutions with different osmoprotectant concentration, ranging from 0.7 to $3.5 \mathrm{M}$. In addition three simulations of water-taurine solutions with the same osmolyte concentration as in the protein simulations were performed, along with a pure water simulation to evaluate the effect of taurine on the solvent in absence of CI2. Each system was energy minimized using the steepest descent method. At first, the solvent alone (and the osmoprotectant when present) was minimized for 1000 steps keeping the protein atoms fixed in their original positions. Next, protein alone (when present) was minimized for 1000 steps. Finally, the whole system was minimized for further 1000 steps. Subsequently, it was equilibrated for 100 ps of MD in the NVT ensemble, followed by further 100 ps at NPT conditions. The productive MD was then run in the NTP ensemble for $11 \mathrm{~ns}$. All simulations were carried out using the GROMACS package ${ }^{7,8}$, version 3.3 and the OPLS force field ${ }^{9}$. The TIP3P water model ${ }^{10}$ was used. Temperature $(448 \mathrm{~K})$ and pressure $(1 \mathrm{~atm})$ were held constant via a weak coupling with an external bath ${ }^{11}$. Bond lengths were fixed to their equilibrium values using the LINCS algorithm ${ }^{12}$ and a 2 fs time step was employed, saving snapshots every 0.2 ps for analysis. The use of bond freezing in MD simulations allows a longer integration time step and is well documented in protein simulations at high temperature ${ }^{13-15}$. The LINCS algorithm was preferred over SHAKE because of its greater stability ${ }^{12}$. Particle Mesh Ewald method ${ }^{16}$ was used for evaluating long range electrostatic interactions, along with a $0.8 \mathrm{~nm}$ cutoff for all nonbonded interactions. The average values of some parameters have been calculated in the first and in the last nanosecond of the productive phase of the simulations. They are referred as "initial" and "final values". Radial distribution functions were calculated over the last $3 \mathrm{~ns}$ of each trajectory.

Trajectories were analysed with special regard to protein and solvent structure, as well as to their mutual interactions. A visual inspection of the protein structure during the trajectories computed in pure water and in the most concentrated taurine system (Fig. 2) evidently displays the different behaviour of the protein in the two cases, with a large unfolding of CI2 in the pure water system and an evident structure preservation in the presence of taurine, which shows a fairly protective effect. On 
a more quantitative ground, the $\mathrm{C} \alpha$ root mean square deviation (RMSD) with respect to the crystal structure was analysed. Comparison of the control trajectory in water with the simulations containing taurine molecules (Fig.3) clearly shows a protective effect in presence of taurine. The effect is also positively correlated with the increasing concentration of the osmoprotectant. The total RMSD values for the two most concentrated simulations of taurine are comparable. However an inspection of the $\mathrm{C} \alpha$ RMSD computed for each residue (Fig.4) evidences a generally stronger protecting effect of the higher concentrated system on the secondary structure elements. This is particularly evident for the residues forming the $\alpha$-helix (ranging from 12 to 24 ). The observed effect parallels the protecting behaviour of the TMAO towards the denaturing effects of urea on the CI2 protein ${ }^{3}$. As the first step in the CI2 unfolding consists in an expansion of the molecule and a destruction of the hydrophobic core $^{4}$, some distances between hydrophobic residues, representative of the geometry of the hydrophobic core, were monitored (Table 1). The control trajectory shows generally increased distances among the hydrophobic residues, while the simulations with the taurine molecules indicate a more retained structure of the hydrophobic core. Also in this case the effect is positively correlated with the increasing concentration of taurine in the solutions. The analysis of the hydrogen bonds (Table 2) yielded more insight in the protein-solvent interplay. In the control trajectory the number of hydrogen bonds between protein and water increases while the number of intramolecular bonds decreases, as an effect of the hydrophobic core breakdown. The presence of the taurine molecules has a stabilizing effect on the number of hydrogen bonds between the protein and the water solvent; the stabilisation in the number of hydrogen bonds is more evident with the increasing concentration of the osmoprotectants. Comparing the number of hydrogen bonds of taurine and water molecules with the protein related to the total number of taurine and water molecules in the system, we have not observed an exclusion of the osmoprotectant molecules from the protein surface as proposed in some papers ${ }^{17}$, suggesting that this behaviour could not be a general feature of all the osmoprotectants. As the importance of hydrogen bonds in protein folding is well documented and has been recently outlined, for example, by the experimental work of Wang and Fitzgerald ${ }^{18}$, we looked at the temporal 
behaviour of the intramolecular (i.e. within the protein) hydrogen bond-lengths distribution during the simulation. The behaviour is sharply influenced by the absence or presence of taurine. In the control trajectory the distribution shifts toward shorter bond-lengths (Fig. 5a) as the trajectory evolves. Remembering that at the same time some bonds are lost (Tab. 2), this indicates that the longest and weakest hydrogen bonds are lost during the unfolding process, so resulting in a strong effect on the secondary and tertiary structure of the protein. In the presence of taurine the distribution of bond lenghts remains instead substantially inalterated in the course of the simulation (Fig. 5b) confirming the greatest stability of the protein in the presence of the osmoprotectant.

The presence of the osmoprotectant can also give rise to indirect protective effect on the protein, inducing a more structured water solvent, that would be then less inclined to attack the protein hydrophobic core resulting in a stabilization of the native structure of the protein.

This effect can be measured from the analysis of the oxygen-oxygen radial distribution function RDF for water $\left(\mathrm{g}(\mathrm{r})_{\mathrm{OO}}\right)$. The height of the first peak, located at $0.28 \mathrm{~nm}$, which is indicative of the tetrahedrally oriented water molecules, increases with the concentration of taurine (Fig. 6), showing that the water becomes effectively more structured. The same behaviour is observed also in the simulations of taurine solutions (in the absence of the protein) confirming that the ordering of the water molecules is an effect induced by the presence of osmoprotectant alone (data not shown). It is interesting to observe that the RDF of the most concentrated solution, resemble very closely the RDF computed at room temperature $(298 \mathrm{~K})$ for a system constituted only by water and protein, where the protein remains stable. The latter RDF is almost coincident with that computed for pure water (i.e. in the absence of the protein) at 298 K.The most ordered solvent structure induced by the taurine molecules is positively correlated with the increased stability of the native structure of the protein. In fact we observe that the deviation of the protein alpha carbons RMSD with respect to the crystal stucture decreases with increasing taurine concentration (Fig.3) and parallely the g(r) OO $_{\text {for }}$ water becomes more peaked (Fig.6). At the molecular level the unfouvaourable unfolding process in the presence of taurine molecules can be explained in the following way. The unfolding process would be 
characterized by the establishment of new interactions of the core region residues, which would become exposed, with the water molecules. This process would require to pay a penalty to destroy the more ordered water structure induced by the taurine molecules.

This ordering effect has been previously reported in simulations of solutions of glycine betaine, another well known osmoprotecting agent ${ }^{19}$. The opposite mechanism was observed in the simulation of the denaturation of CI2 caused by the urea molecules ${ }^{3}$. The addition to the latter system of TMAO has evidenced a protecting effect on the protein, once again accompanied by an increased ordered structure of the solvent.

In conclusion, simulations of taurine solutions have been carried at different concentrations in the presence or absence of $\mathrm{CI} 2$. The protective effect of the taurine, positively correlated with the increasing concentration of the osmoprotectant, has been analysed in terms of the stability of the overall structure of the protein and of its secondary structure elements. The molecular picture that emerges from the analysis of the simulations, indicates that taurine molecules induce an ordering in the structure of the water molecules which are so less prone to attack the residues which would become exposed due to the first steps of the unfolding process. Also the hydrophobic core of the CI2 remains substantially more stable in the presence of the taurine molecules, as evidenced by the behaviour of the distances between some key residues.

The results obtained modelling the action of taurine against thermal denaturation parallel those previously obtained in the simulation of the protective effect of TMAO toward the chemical denaturation of CI2 induced by urea.

\section{Aknowledgments}

We thank Dott. Giuseppe Lanzani for the parallel implementation of our PC-Linux cluster. 
Bibliography

${ }^{1}$ G.N. Somero, in Water and Life, Ed. G.N. Somero, C. B. Osmund, C. L. Bolis, Springer, Berlin, 1992.

${ }^{2}$ P. H. Yancey, M. E. Clark, S. C. Hand, R. D. Bowlus, G. N. Somero, Science 217 (1982) 1214.

${ }^{3}$ B.J. Bennion, V. Daggett, Proc. Natl. Acad. Sci. USA 101 (2004) 6433.

${ }^{4}$ V. Daggett, Chem. Rev. 106 (2006) 1898.

${ }^{5}$ R. Day, B. J. Bennion, S. Ham, V. Daggett, J. Mol. Biol., 322 (2002) 189.

${ }^{6}$ Y. Harpaz, N. Elmasry, A. R. Fersht, K. Henrick, Proc. Natl. Acad. Sci. USA, 91 (1994) 311.

${ }^{7}$ H.J.C. Berendsen, D. van der Spoel, R. van Drunen, Comp. Phys. Comm., 91 (1995) 43.

${ }^{8}$ E. Lindahl, B. Hess, D. van der Spoel, J. Mol. Mod., 7 (2001) 306.

${ }^{9}$ W.L. Jorgensen, D.S. Maxwell., and Tirado-Rives J., J. Am. Chem. Soc., 118 (1996) 11225.

${ }^{10}$ W.L. Jorgensen, J. Chandrasekhar, J. D. Madura, R. W. Impey, M. L. Klein, J. Chem. Phys., 79 (1983) 926.

${ }^{11}$ H. J. C. Berendsen, J. P. M. Postma., van Gunsteren W. F., DiNola A., and Haak J. R., J. Chem. Phys., 81 (1984) 3684.

12 B. Hess, H. Bekker, H. J. C. Berendsen, J. Fraaije, J. Comput. Chem., 18 (1997) 1463.

${ }^{13}$ A. S. N. Seshasayee, Theoretical Biology and Medical Modelling, 2 (2005).

${ }^{14}$ M.M. Seibert, A. Patriksson, B. Hess, D. Van der Spoel, J. Mol. Biol, 354 (2005) 173.

15 X. Daura, W.F. Van Gunsteren, D. Rigo, B. Jaun, D. Seebach, Chem. Eur. J. 3 (1997) 1410.

${ }^{16}$ T. Darden, D. York, L. Pederson, J. Chem. Phys., 98 (1993) 10089.

${ }^{17}$ T. Arakawa, R. Bhat, S. Timasheff, Biochemistry, 29 (1990) 1914.

${ }^{18}$ Wang M., Wales T.E. and Fitzgerald M.C., Proc. Natl. Acad. Sci. USA, 103 (2006) 2600.

${ }^{19}$ M. Civera, A. Fornili, M. Sironi, S.L. Fornili, Chem. Phys. Lett., 367 ( 2003) 238.

${ }^{20}$ B.J. Bennion, V. Daggett, Proc. Natl. Acad. Sci. USA, 100 (2003) 5142.

${ }^{21}$ W. Humphrey, A. Dalke, K. Schulten, J. Molec. Graphics, 14 (1996) 33. 
Figures

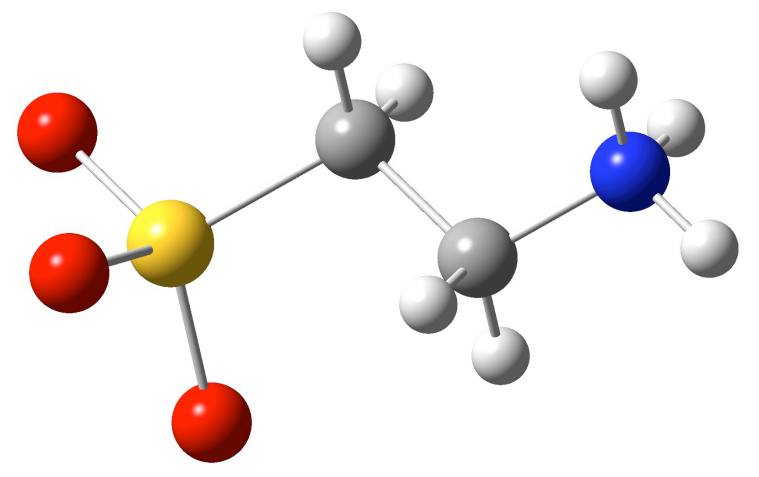

Fig.1

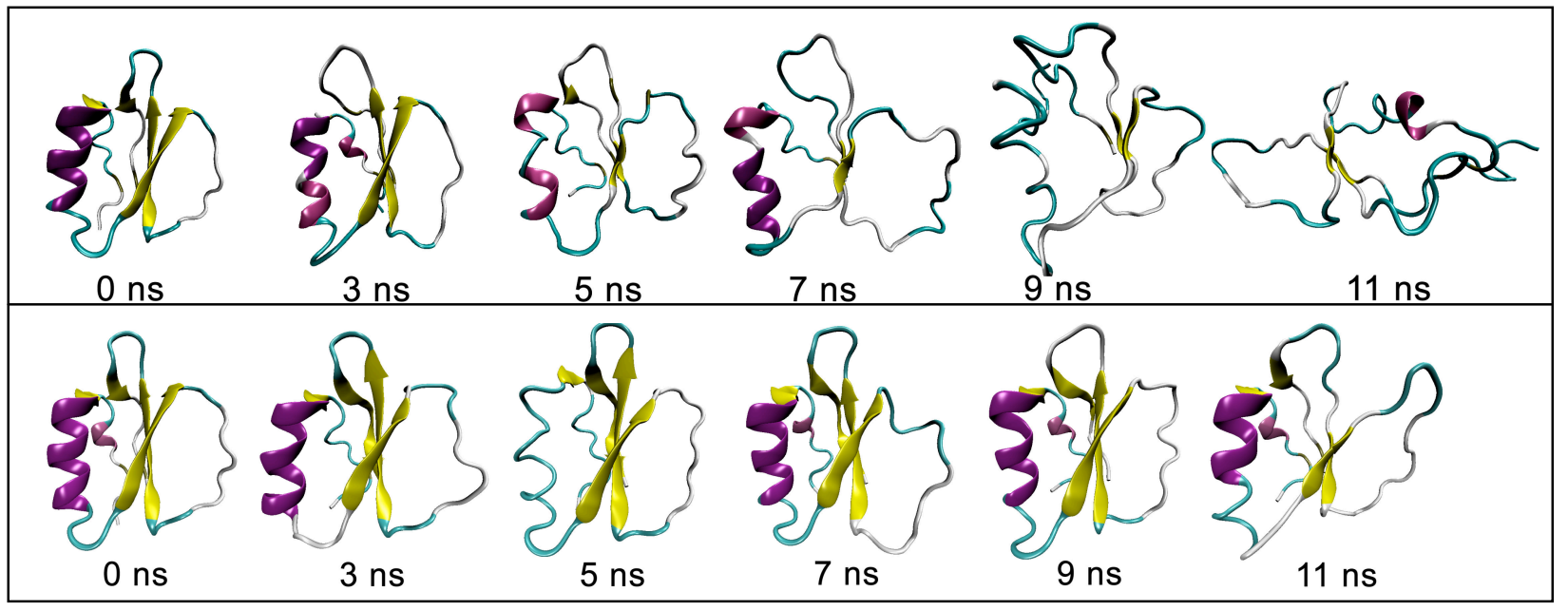

Fig.2 


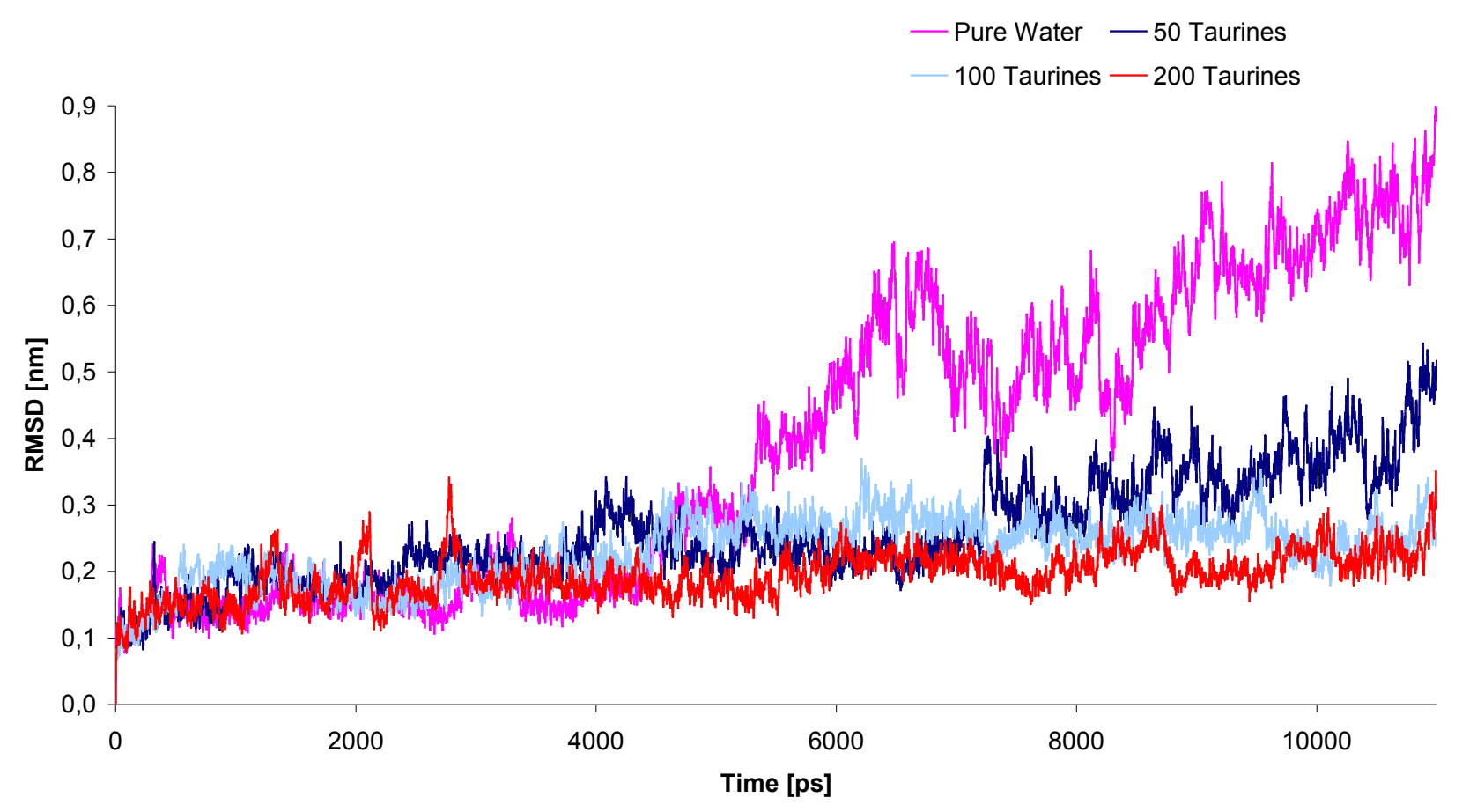

Fig.3

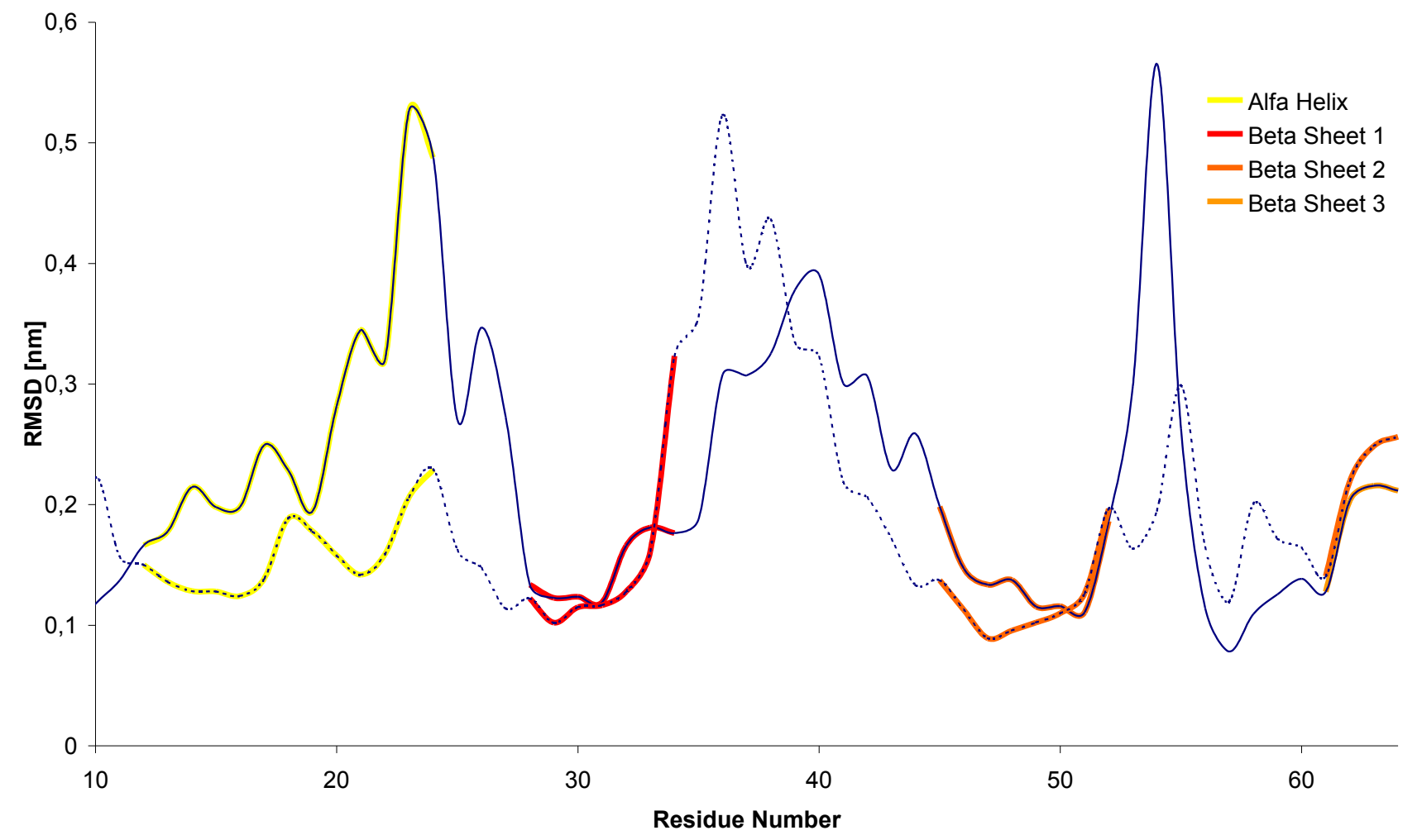




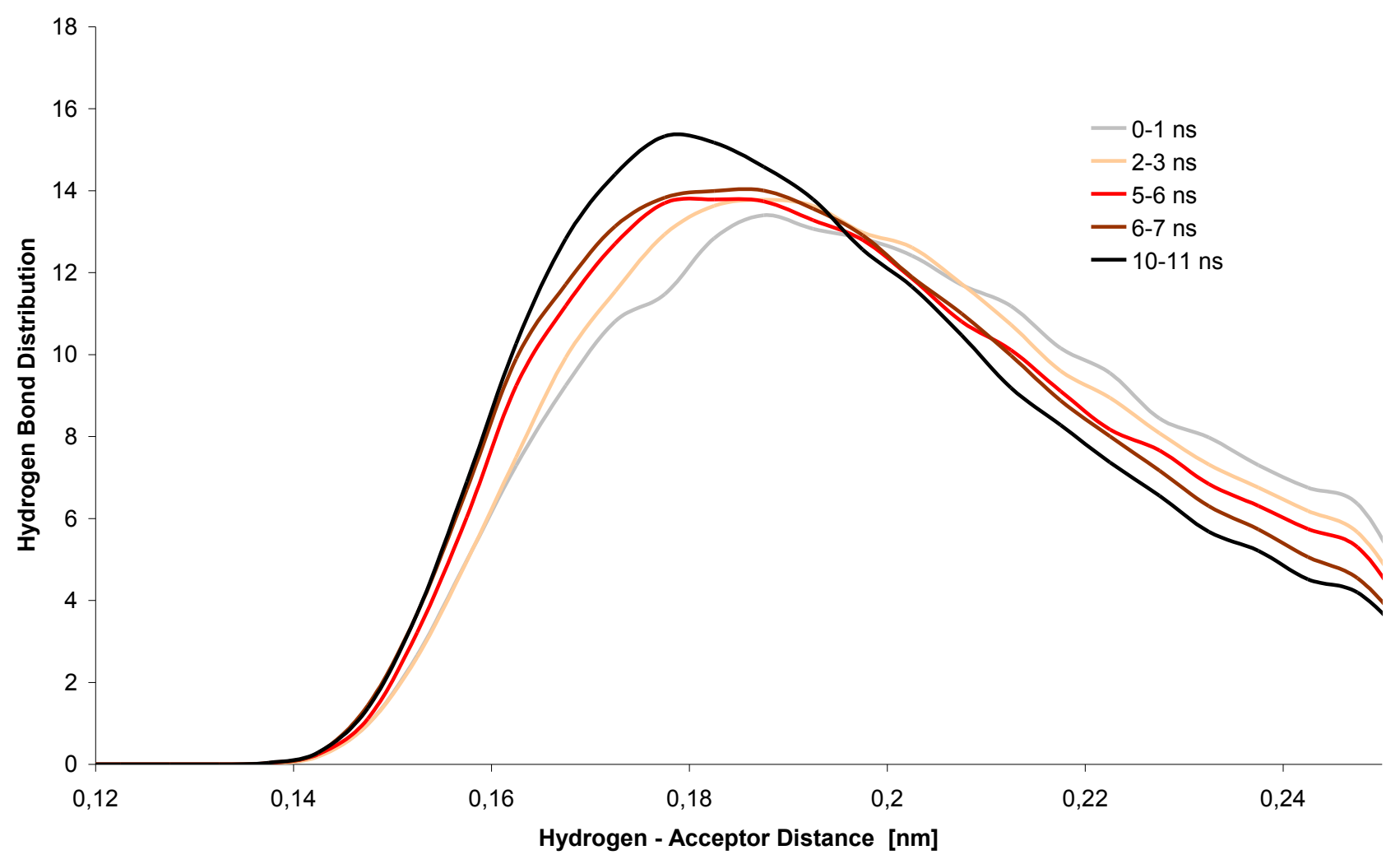

Fig.4

Fig.5a 


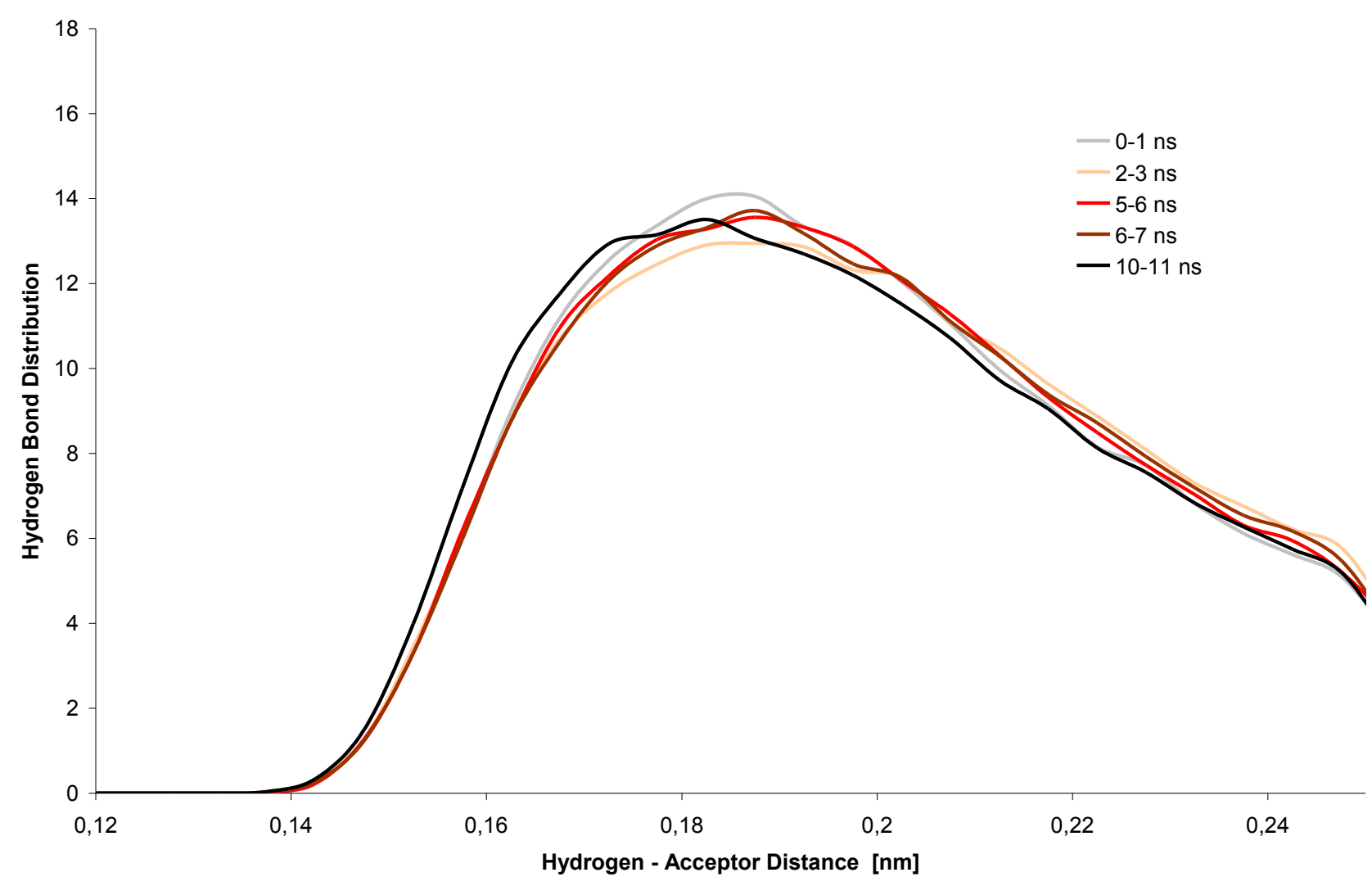

Fig. $5 b$ 


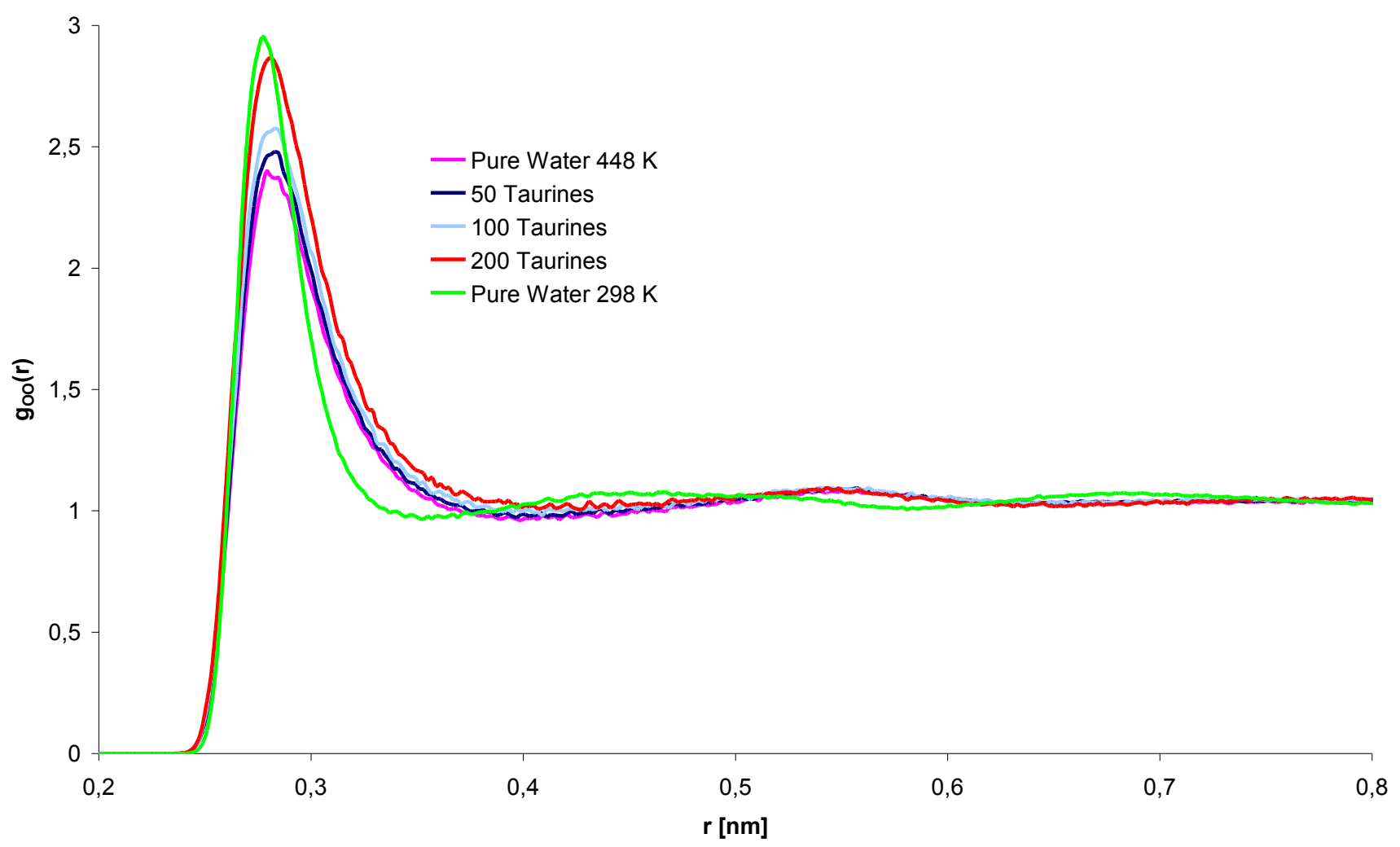

Fig.6

Figure Captions 
Fig.1 The structure of 2-aminoethansulphonic acid (taurine) molecule.

Fig.2 Comparative behaviour of CI2 in the control simulation (upper panel) and in presence of 200 taurine molecules (lower panel). The images were drawn with VMD $1.8 .3^{21}$.

Fig.3 RMSD deviation of CI2 from the crystal structure during the different simulations.

Fig.4 Average per residue RMSD monitored during the last 3 ns of the simulations of CI2 with 100 taurine (continuous line) and 200 taurine (dotted line).

Fig.5a,b Temporal behaviour of the intramolecular hydrogen bond distribution of CI2 during the simulation in pure water (a) and in the most concentrated taurine system (200 taurines).

Fig.6 Radial distribution function of water oxygen-oxygen atoms for the different MD simulations.

Tables 


\begin{tabular}{|c|c|c|c|c|c|c|c|c|c|c|}
\hline & & \multicolumn{3}{|c|}{ ILE20-TRP5 } & \multicolumn{3}{|c|}{ LEU49-ALA16 } & \multicolumn{3}{|c|}{ TRP5-ALA16 } \\
\hline \multirow{2}{*}{ Control } & initial & 0.61 & $\hat{1}$ & 112 & 0.84 & $\hat{1}$ & 0 & 0.89 & $\hat{A}$ & 064 \\
\hline & final & 1.74 & $\Delta$ & 1.13 & 1.00 & $\Delta$ & 0.16 & 1.52 & $\Delta$ & 0.64 \\
\hline \multirow{2}{*}{50 TAU } & initial & 0.63 & \multirow{2}{*}{$\Delta$} & \multirow{2}{*}{0.92} & 0.84 & \multirow{2}{*}{$\Delta$} & \multirow{2}{*}{0.26} & 0.88 & \multirow{2}{*}{$\Delta$} & \multirow{2}{*}{0.52} \\
\hline & final & 1.55 & & & 1.09 & & & 1.40 & & \\
\hline \multirow{2}{*}{$100 \mathrm{TAU}$} & initial & 0.69 & \multirow{2}{*}{$\Delta$} & \multirow{2}{*}{0.15} & 0.86 & \multirow{2}{*}{$\Delta$} & \multirow{2}{*}{-0.04} & 0.89 & \multirow{2}{*}{$\Delta$} & \multirow{2}{*}{0.00} \\
\hline & final & 0.84 & & & 0.82 & & & 0.89 & & \\
\hline \multirow{2}{*}{200 TAU } & initial & 0.64 & \multirow{2}{*}{$\Delta$} & \multirow{2}{*}{0.10} & 0.86 & \multirow{2}{*}{$\Delta$} & \multirow{2}{*}{0.00} & 0.95 & \multirow{2}{*}{$\Delta$} & \multirow{2}{*}{0.13} \\
\hline & final & 0.74 & & & 0.86 & & & 1.08 & & \\
\hline
\end{tabular}

Tab.1 Evolution of the distance (expressed in $\mathrm{nm}$ ) between the centres of mass of some residues representative of the hydrophobic core during the different simulations.

\begin{tabular}{|c|c|c|c|c|c|c|c|c|c|c|c|c|}
\hline & \multicolumn{3}{|c|}{ Control } & \multicolumn{3}{|c|}{50 TAU } & \multicolumn{3}{|c|}{100 TAU } & \multicolumn{3}{|c|}{200 TAU } \\
\hline & initial & final & $\Delta n$ & initial & final & $\Delta n$ & initial & final & $\Delta n$ & initial & final & $\Delta n$ \\
\hline Prot $-\mathrm{H}_{2} \mathrm{O}$ & 103 & 115 & 12 & 99 & 105 & 7 & 91 & 97 & 6 & 87 & 85 & -2 \\
\hline Prot - Prot & 39 & 34 & -5 & 39 & 36 & -3 & 40 & 37 & -3 & 39 & 37 & -2 \\
\hline Prot - Tau & -- & -- & -- & 3 & 4 & 1 & 7 & 8 & 1 & 14 & 14 & 0 \\
\hline
\end{tabular}

Tab.2 Evolution of the number of H-bonds between the different components of the simulated systems. H-bonds require a donor-acceptor distance not greater than $0.35 \mathrm{~nm}$ and an acceptor-donor hydrogen angle not greater than 30 degrees. 